\title{
Comparative Study of Ordinary and Supa Sections of Cold Formed Steel
}

\author{
Drishya S Krishna \\ Post Graduate student, Department of Civil Engineering \\ Sree Narayana Gurukulam College of Engineering \\ Ernakulam, Kerala, India
}

\author{
Binu P \\ Assoc. Professor, Department of Civil Engineering \\ Sree Narayana Gurukulam College of Engineering \\ Ernakulam, Kerala, India
}

\begin{abstract}
Cold-formed steel (CFS) is one of the types of steel which is processed by cold-working procedures. It is carried out near the room temperature. They are generally used as flexural members in light weight steel construction. They are structurally and costly effective, thus their thin walled members are used in low and mid-rise building construction. There was a rapid growth in the usage of CFS in buildings because, several innovative and structurally efficient CFS products were easily available. Their advantages include lightness, high strength and stiffness, ease of prefabrication and mass production, fast and easy erection and installation etc. Ordinary and Supa are the two types of cold formed steel sections. The Supa section is an innovative section introduced in Australia. It is having the specialty of unique ribbed web and curved lip elements thus it provides superior flexural strength when compared with traditional channel sections. This paper presents the comparison of ordinary and supa sections on the basis of performance of both the sections. $\mathrm{C}, \mathrm{Z}$ and sigma sections of both ordinary and Supa sections are numerically evaluated using buckling analysis. It was found that Supa sections were effective than the ordinary sections of cold formed steel.
\end{abstract}

Keywords-Flexural members; ribbed web; curved lip.

\section{INTRODUCTION}

In steel construction, there are two main families of structural members. One is the familiar group of hot-rolled shapes and members built-up of plates. The other, less familiar but of growing importance, is composed by coldformed sections from sheets, strips, plates or flat bars in roll forming machines, by press-brake or bending brake operations [1]. These are cold-formed steel structural members. The thickness of steel sheets generally used in cold-formed steel structural members ranges from $0.5 \mathrm{~mm}$ to $4 \mathrm{~mm}$ for sheeting and from $1 \mathrm{~mm}$ to $8 \mathrm{~mm}$ for profiles, respectively [1]. Steel plates and bars as thick as $25 \mathrm{~mm}$ can be cold-formed successfully into structural shapes. The use of cold-formed steel members in building construction began in the 1850s in both United States and Great Britain [1].

In general, cold-formed steel structural members provide the following advantages in building construction [1]:

1. As compared with thicker hot-rolled shapes, cold-formed light members can be manufactured for relatively light loads and/or short spans.

2. Unusual sectional configurations can be produced economically by cold-forming operations and consequently favorable strength-to-weight ratios can be obtained.
3. Nestable sections can be produced, allowing for compact packaging and shipping.

4. Load carrying panels and decks can provide useful surfaces for floor, roof, and wall construction.

5. Load-carrying panels and decks not only withstand loads normal to their surfaces, but they can also act as shear diaphragms to resist force in their own planes if they are adequately interconnected to each other and to supporting members [1].

Cold formed steel is used as flexural members in light weight steel construction. They are structurally and costly effective, thus their thin walled members are used in low and mid-rise building construction. There was a rapid growth in the usage of CFS in buildings because, several innovative and structurally efficient CFS products were easily available. Advantages also include lightness, high strength and stiffness, ease of prefabrication and mass production, fast and easy erection and installation etc. Products of cold formed steel are available in different shapes and sizes. So, it is effectively used as partition walls, roof purlins, roof trusses, floor joists. Ordinary and Supa are the two types of cold formed steel sections. The Supa section is an innovative section introduced in Australia. It is having the specialty of unique ribbed web (crimps) and curved lip elements thus it provides superior flexural strength when compared with traditional channel sections. This paper presents the comparison of ordinary and supa sections on the basis of performance of both the sections. Performance can be effectively analyzed by the load carrying capacity of each section. Cee, Zed and Sigma sections of both ordinary and supa are taken for the analysis. That is Cee, Zed and Sigma sections of ordinary cold formed steel are compared with that of supa sections respectively.

\section{NUMERICAL ANALYSIS}

Dimensions of the model and other relevant input parameters are taken from a journal to verify the accuracy of a numerical model. Finite element software is used to reciprocate the numerical analysis from the particular journal. The details were taken from Journal [5] for the validation of mathematical model using the finite element software ANSYS 16.1. The model named as ETF-SC20015 was taken for the purpose of validation. The model is having ribbed web and curved lips. Section depth is taken as $204.9 \mathrm{~mm}$, flange width as $69.7 \mathrm{~mm}$, corner radius as $5 \mathrm{~mm}$, lip width as $22.9 \mathrm{~mm}$ 
and $12 \mathrm{~mm}$ stiffeners. Elastic modulus and Poisson's ratio of steel from the journal are $200,000 \mathrm{MPa}$ and 0.3 respectively. Modelling of the specimen as per the journal and meshing were done using the software. Modelled specimen is loaded so that displacements and forces were obtained. Loaddeflection curve of the model is obtained. The web crippling capacity obtained from the journal was $3.04 \mathrm{kN}$ and that obtained from FEA is $2.849 \mathrm{kN}$. Hence, the percentage error is less than $10 \%$. Fig. 1 shows the supacee section used for the validation.

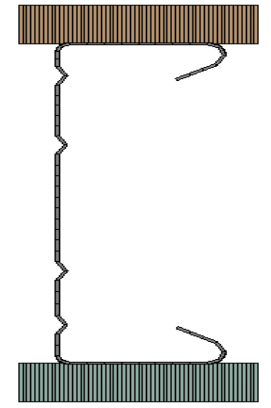

$\stackrel{t}{\longrightarrow} x$

Fig. 1. SupaCee Section

Buckling analysis is done in this paper for all the sections of ordinary and supa to study their performance and to identify which one is better.

\section{A. Modelling of ordinary and supa sections}

Numerical modeling of the OrdinaryCee, OrdinaryZed, OrdinarySigma, SupaCee, SupaZed and SupaSigma models were performed by the FE software ANSYS 16.1 WORKBENCH. Fig. 2 and 3 shows the modelled view of OrdinaryCee and SupaCee respectively.

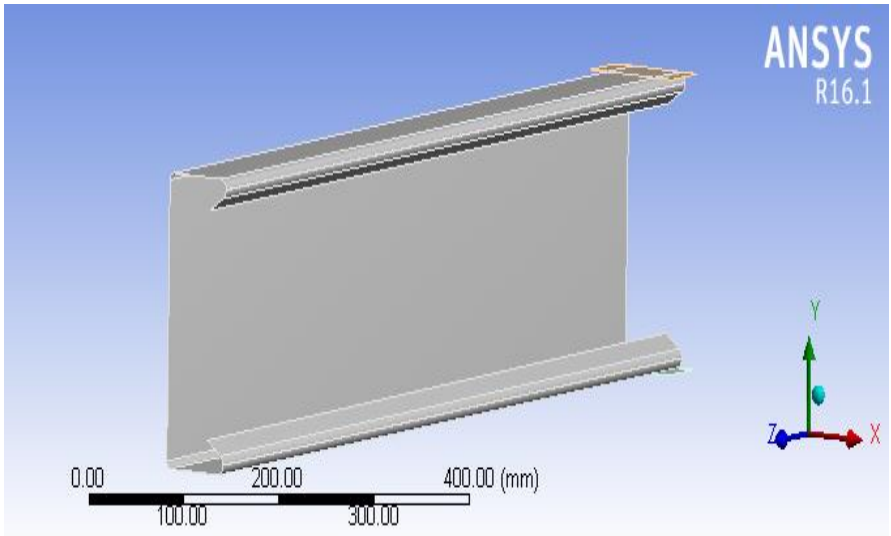

Fig. 2. Modelled view of OrdinaryCee

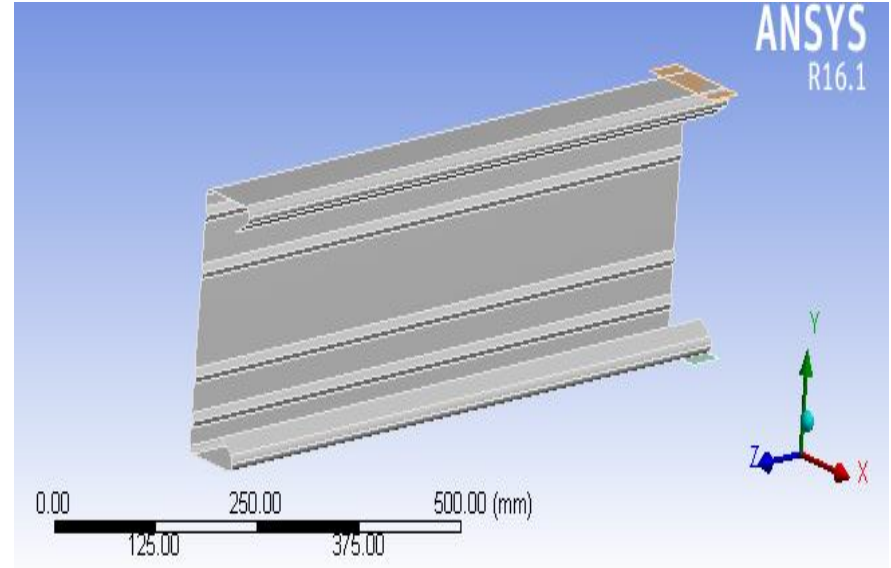

Fig.3. Modelled view of SupaCee

Material properties of the models were chosen from the engineering data of the ANSYS software. Pre-assigned values for each material is given in the engineering data section of the software. Table. 1 shows the material properties of the model.

TABLE I. Material properties of models

\begin{tabular}{|c|c|}
\hline Material & Cold formed steel \\
\hline $\begin{array}{c}\text { Modulus of } \\
\text { Elasticity }\end{array}$ & $2 \times 10^{5} \mathrm{~N} / \mathrm{mm}^{2}$ \\
\hline Poisson's Ratio & 0.3 \\
\hline Density & $7850 \mathrm{~kg} / \mathrm{m}^{3}$ \\
\hline Yield strength & $250 \mathrm{MPa}$ \\
\hline
\end{tabular}

Totally six sections were modelled. Nomenclature for the model is given as per the presence or absence of crimps in the web and shape of the model. The models without crimps in the web were named as OrdinaryCee, OrdinaryZed and OrdinarySigma. The models with crimps in the web were named as SupaCee, SupaZed and SupaSigma. OrdinaryCee and SupaCee is having the shape of letter C. OrdinaryZed and SupaZed is having the shape of letter Z. OrdinarySigma and SupaSigma is having the shape of symbol sigma.

\section{B. Support and loading}

Support condition and loading was given to all the models after modelling. The fixed support was given at the bottom of the model and displacement was given at the top of the model. Fig. 4 shows the SupaCee model with fixed support at the base. Fig. 5 and 6 shows fixed support of SupaZed and SupaSigma respectively. Six models were obtained and among that three of them belongs to ordinary section which is not having crimps in their web and other three belongs to the supa section which is having crimps in their web. 


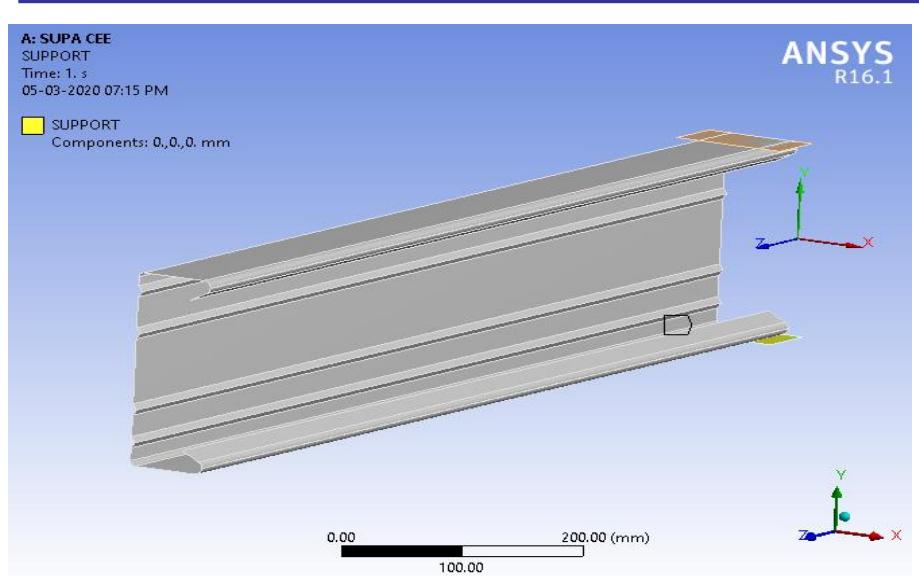

Fig. 4. SupaCee model with fixed support

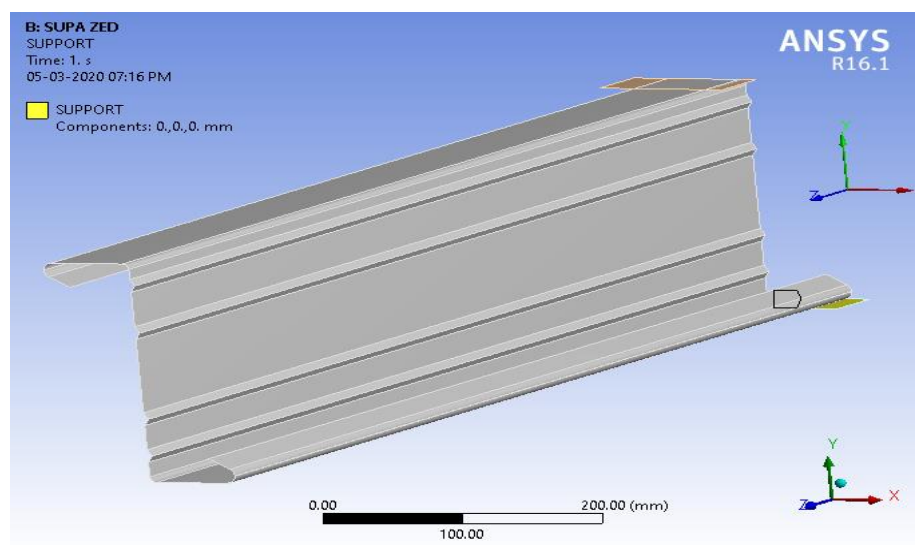

Fig. 5. SupaZed model with fixed support

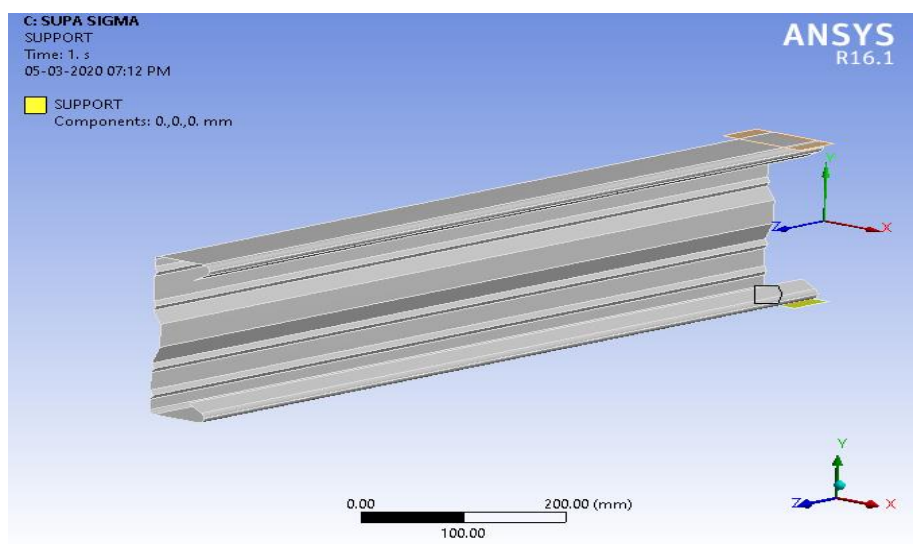

Fig. 6. SupaSigma model with fixed support

Buckling analysis was done to all the sections using the software ANSYS 16.1 WORKBENCH. After the analysis, the buckling behavior of all the models were obtained using the load-deflection curve by taking the values from ANSYS. The performance of OrdinaryCee is compared with SupaCee, OrdinaryZed with SupaZed and OrdinarySigma with SupaSigma. Load carrying capacity of each section was considered to find the best section and for comparison. Stiffness of each section is found out using total deformation $(\mathrm{mm})$ and force reaction $(\mathrm{kN})$.

\section{RESULTS AND DISCUSSION}

Buckling analysis was done to the six sections such as OrdinaryCee, OrdinaryZed, OrdinarySigma, SupaCee, SupaZed and SupaSigma. Values of total deformation and force reaction were taken from the ANSYS software and these values were used to plot the load-deflection curve of all the sections. Figure 7 shows the load-deflection curve of SupaCee, SupaZed and SupaSigma. Figure 8 shows the Loaddeflection graph of OrdinaryCee, OrdinaryZed and OrdinarySigma. Table 2 shows the maximum deformation and corresponding force reaction of all the sections.

\section{LOAD-DEFLECTION GRAPH}

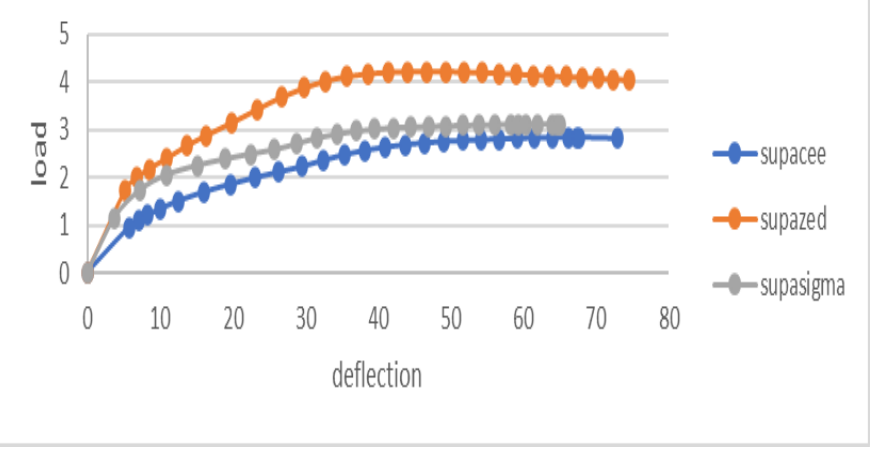

Fig. 7. Load deflection graph of SupaCee, SupaZed, SupaSigma

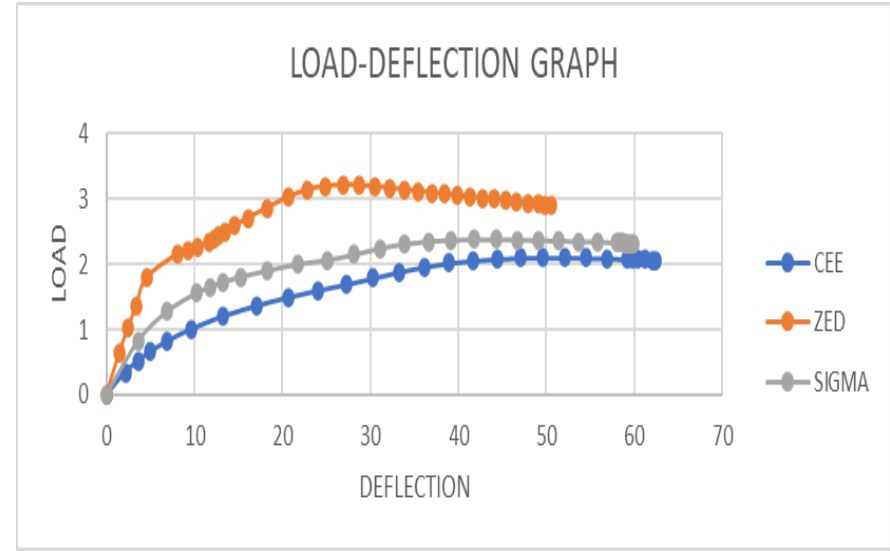

Fig. 8. Load-deflection graph of OrdinaryCee, OrdinaryZed and OrdinarySigma

Table II. Maximum deformation and force reaction of all sections

\begin{tabular}{|c|c|c|}
\hline Section & $\begin{array}{c}\text { Max deformation } \\
(\mathbf{m m})\end{array}$ & Force reaction(kN) \\
\hline SupaCee & 67.629 & 2.849 \\
\hline SupaZed & 46.71 & 4.2269 \\
\hline SupaSigma & 64.541 & 3.1266 \\
\hline OrdinaryCee & 49.59 & 2.1042 \\
\hline OrdinaryZed & 26.86 & 3.2086 \\
\hline OrdinarySigma & 41.764 & 2.3765 \\
\hline
\end{tabular}

Fig 7 and 8 shows that up to a particular deformation, the value of force reaction is increasing and then decreasing for all the sections. At that point, failure is occurred. From the values of table 2, stiffness was found to be more for the 
ordinary sections of cold formed steel. But when considering the load carrying capacity, ordinary sections are less effective than the supa sections. From the figure 7 and 8 it is evident that load carrying capacity of all the supa sections are higher than that of the ordinary sections. On comparison of each sections it was seen that SupaCee is having 35.4\% more load carrying capacity than OrdinaryCee, SupaZed is having $31.7 \%$ more load carrying capacity than OrdinaryZed and SupaSigma is having $31.5 \%$ more load carrying capacity than the OrdinarySigma. Hence, the strength of all the supa sections will be greater that that of the ordinary sections of cold formed steel. SupaZed is found to be the best among all other sections which is having the capacity to carry load up to $4.2269 \mathrm{kN}$.

From Fig. 7 it is observed that, SupaZed is having more load carrying capacity than SupaCee and SupaSigma. But when considering yielding capacity SupaCee and SupaSigma is more effective. Similarly, from Fig. 8 it is observed that, OrdinaryZed is having more load carrying capacity than OrdinaryCee and OrdinarySigma. But when considering yielding capacity OrdinaryCee and OrdinarySigma is more effective.

\section{CONCLUSION}

In this research, behaviour of all the models were analysed in ANSYS WORKBENCH 16.1 using buckling analysis. Compared the performance of SupaCee with OrdinaryCee, SupaZed with OrdinaryZed and SupaSigma with OrdinarySigma. Change in the properties of ordinary models were due to the absence of crimps in their web. Hence, strength of each Supa sections was greater than that of the ordinary sections. In this paper it is observed that, SupaCee is having $35.4 \%$ more load carrying capacity than OrdinaryCee, SupaZed is having $31.7 \%$ more load carrying capacity than OrdinaryZed and SupaSigma is having $31.5 \%$ more load carrying capacity than the OrdinarySigma. SupaZed was found to be the best section.

\section{REFERENCES}

[1] Ludovic Alexander, Nagy Zsolt, "Cold Formed Steel Structures for Residential and Non-Residential buildings," Article, pp. 1-11, January 2000.

[2] Keerthan P, Mahen Mahendran and Edward Steau, "Experimental study of web crippling behaviour of hollow flange channel beams under two flange load cases," Article, pp. 207-219, August 2014.

[3] Natario P, N.Silvestre, D.Camotim, "Web crippling of beams under ITF loading: A novel DSM-based design approach," Article, pp. 812-824, October 2016.

[4] Prabakaran K and Schuster R. M, "Web Crippling of Cold Formed Steel Members," International Specialty Conference on ColdFormed Steel Structures, pp. 151-164, October 1998.

[5] Sundararajah L, Mahen Mahendran, Poolaganathan Keerthan, "Web crippling studies of SupaCee sections under two flange load cases," Article, pp. 582-587, June 2017.

[6] Sundararajah L, "Web Crippling Studies of Cold-formed Steel Channel Beams," Thesis, pp. 1-40, 2017.

[7] Sundararajah L, Mahen Mahendran, Poolaganathan Keerthan, "Design of SupaCee Sections Subject to Web Crippling under OneFlange Load Cases," Journal of Strucural Engineering, pp.1-12, 2018. 